Draft Version November 4, 2014

Preprint typeset using LTEX style emulateapj v. 08/22/09

\title{
KINEMATICS OF THE OUTFLOW FROM THE YOUNG STAR DG TAU B: ROTATION IN THE VICINITIES OF AN OPTICAL JET
}

\author{
Luis A. Zapata ${ }^{1}$, Susana Lizano ${ }^{1}$, Luis F. Rodríguez ${ }^{1}$, Paul T. P. Ho ${ }^{2,3}$, Laurent Loinard $^{1}$, \\ Manuel Fernández-LóPEZ ${ }^{3}$, ANd DANIEl TAFOYA ${ }^{1}$ \\ Draft version November 4, 2014
}

\begin{abstract}
We present ${ }^{12} \mathrm{CO}(2-1)$ line and $1300 \mu \mathrm{m}$ continuum observations made with the Submillimeter Array (SMA) of the young star DG Tau B. We find, in the continuum observations, emission arising from the circumstellar disk surrounding DG Tau B. The ${ }^{12} \mathrm{CO}(2-1)$ line observations, on the other hand, revealed emission associated with the disk and the asymmetric outflow related with this source. Velocity asymmetries about the flow axis are found over the entire length of the flow. The amplitude of the velocity differences is of the order of $1-$ $2 \mathrm{~km} \mathrm{~s}^{-1}$ over distances of about $300-400 \mathrm{AU}$. We interpret them as a result of outflow rotation. The sense of the outflow and disk rotation is the same. Infalling gas from a rotating molecular core cannot explain the observed velocity gradient within the flow. Magneto-centrifugal disk winds or photoevaporated disk winds can produce the observed rotational speeds if they are ejected from a keplerian disk at radii of several tens of AU. Nevertheless, these slow winds ejected from large radii are not very massive, and cannot account for the observed linear momentum and angular momentum rates of the molecular flow. Thus, the observed flow is probably entrained material from the parent cloud. DG Tau B is a good laboratory to model in detail the entrainment process and see if it can account for the observed angular momentum.
\end{abstract}

Subject headings: stars: pre-main sequence - ISM: jets and outflows - individual: (DG Tau B) - individual: (Taurus Molecular Cloud)

\section{INTRODUCTION}

Jets and outflows from young stars play a key role in the star-formation process, however their nature is still under debate (Arce et al. 2007). The general consensus is that fast protostellar jets are driven and collimated by rotating magnetic fields anchored to the star-disk system (Pudritz et al. 2007; Shang et al. 2007). However, it is not clear where these magnetic fields are anchored to the disk: it could be from the radius at which the stellar magnetosphere truncates the disc $(X$ wind, Shu et al. 2000) or from an extended region of the disc (disc wind, Königl \& Pudritz 2000). Magneto-hidrodynamic (MHD) models predict that the jet should inherit a toroidal angular momentum component or, in other words, rotation (Fendt 2011). The magnetic field forces the corotation of the gas up to the Alfvèn radius. Therefore, the magnitude and position of these movements within the outflow would give, in principle, information about their origin on the disk (Anderson et al. 2003). We refer the reader to the introduction of Zapata et al. (2010) for details concerning to the previous studies on jet/outflow rotation based on optical, infrared and millimeter studies. In this paper (Zapata et al. 2010), the results and limits on previous studies are discussed.

Located at about $150 \mathrm{pc}$, DG Tau B is a low-luminosity $\left(0.88 \mathrm{~L}_{\odot}\right)$ class $\mathrm{I} / \mathrm{II}$ young star located about $1^{\prime}$ to the $\mathrm{SW}$ of the well-known T Tauri star DG Tau (Jones \& Cohen 1986; Eislöffel \& Mundt 1998; Torres et al. 2009; Luhman et al. 2010; Rodríguez et al. 2012). DG Tau B powers an asym-

\footnotetext{
${ }^{1}$ Centro de Radioastronomía y Astrofísica, UNAM, Apdo. Postal 3-72 (Xangari), 58089 Morelia, Mich., México

${ }^{2}$ Academia Sinica Institute of Astronomy and Astrophysics, Taipei, Taiwan

${ }^{3}$ Harvard-Smithsonian Center for Astrophysics, 60 Garden Street, Cambridge, MA 02138, USA

${ }^{4}$ Astronomy Department, University of Illinois, 1002 West Green Street, Urbana, IL 61801, USA
}

metric bipolar jet (HH 159) mapped at optical/infrared wavelengths (Eislöffel \& Mundt 1998). The red lobe, located to its NW consists of a chain of bright knots extending to about $1^{\prime}$ from the source, while the blue lobe located to its SE is much more fainter and less collimated, and is detected only up to $\sim 10$ " from the star (Mundt \& Fried 1983; Eislöffel \& Mundt 1998; Podio et al. 2011). A molecular outflow mapped in carbon monoxide $(\mathrm{CO})$ is observed principally associated with the NW fast optical jet (Mitchell et al. 1997). These authors found that the $\mathrm{CO}$ outflow is similar to the optical outflow: the $\mathrm{CO}$ redshifted emission extends at least $6000 \mathrm{AU}\left(40^{\prime \prime}\right.$ at the distance of $150 \mathrm{pc}$ ) to the NW of the star, while the blueshifted $\mathrm{CO}$ emission is confined to a compact region, which is less than $500 \mathrm{AU}$ (about $3^{\prime \prime}$ ). McGroarty \& Ray (2004) proposed that the Herbig-Haro objects 836 and 837, located several arcmin to the SE of DG Tau B, could be tracing ejecta from this star that took place $\sim 10^{4}$ years ago.

At the base of the outflow emerging from DG Tau B there is a circumstellar disk placed close to edge-on $\left(\sim 65^{\circ}\right.$; Guilloteau et al. 2011), and detected for the first time in absorption by the broadband imaging of the Hubble Space Telescope (Stapelfeldt et al. 1997). The disk was confirmed by ${ }^{13} \mathrm{CO}$ millimeter observations (Padgett et al. 1999). The source position has been determined from $3.5 \mathrm{~cm}$ VLA radio continuum observations by Rodriguez et al. (1995), who locate it within the observed dark lane. The position is at $\alpha_{J 2000.0}=04^{\mathrm{h}} 27^{\mathrm{m}} 02^{\mathrm{s}} 55, \delta_{J 2000.0}=+26^{\circ} 05^{\prime} 30^{\prime \prime} .7$. The disk has been mapped at millimeter wavelengths using sub-arcsecond resolution with the Plateau de Bure Interferometer (PdBI) (Guilloteau et al. 2011). They found a deconvolved size for the disk of 0 .' $^{\prime} 69 \times 00^{\prime \prime} 34$ with a P.A. $=26^{\circ}$, and a mass of $0.068 \mathrm{M}_{\odot}$. Gramajo et al. (2010) modeled the SED of DG Tau B as a young star with a mass of $0.5 \mathrm{M}_{\odot}$, a temperature of $4000 \mathrm{~K}$ and a radius of $2.5 \mathrm{R}_{\odot}$.

In this paper, we present millimeter line and continuum ob- 


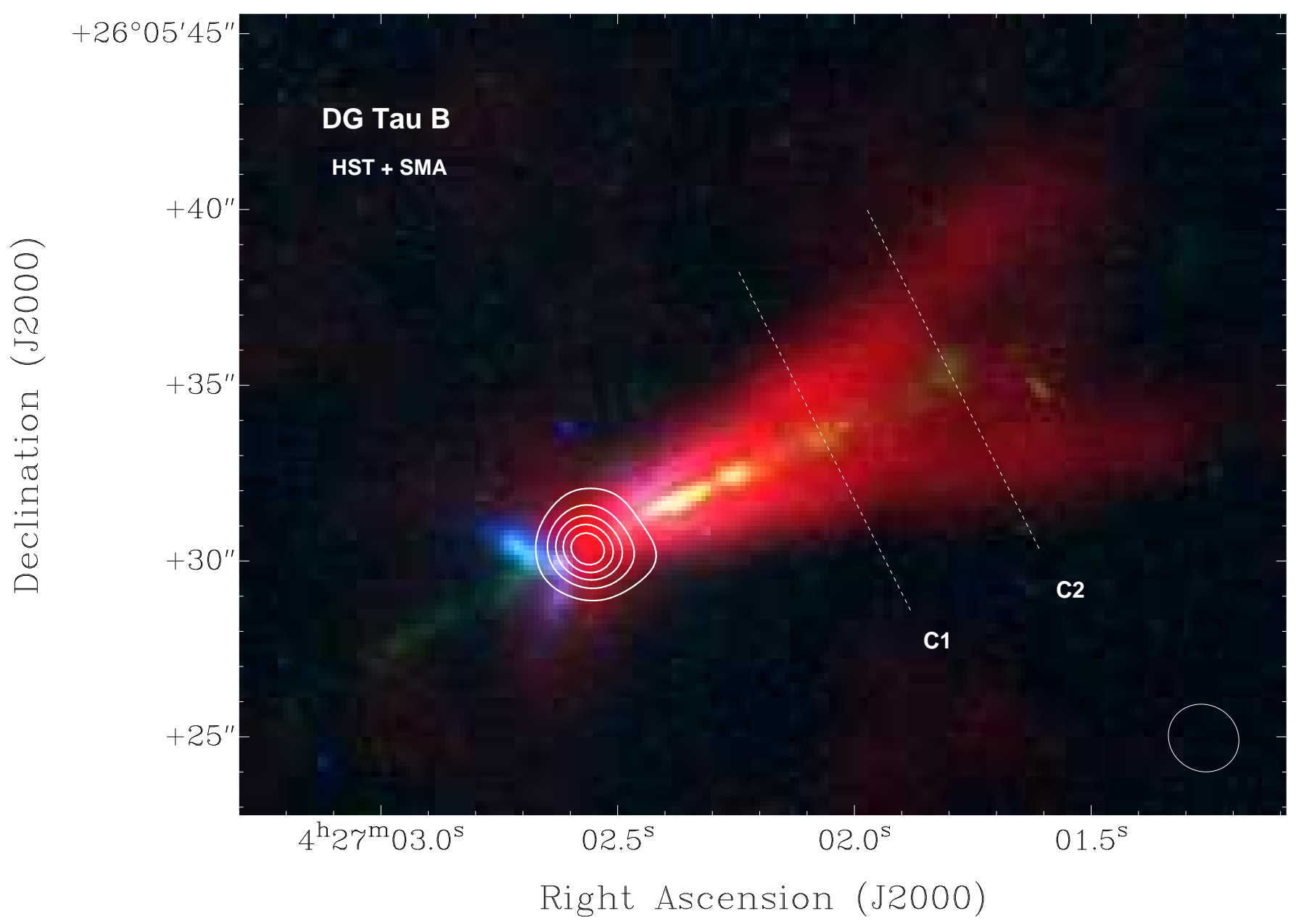

Figure 1. SMA and HST color-coded image of DG Tau B. Color coding: SMA total CO integrated intensity emission (red), HST-WFPC2 images with the spectral filters F814w (blue) and F675w (green). The color-coded image is in addition overlaid with an SMA $1300 \mu m$ continuum contour image obtained by averaging the line-free spectral channels in the upper side band. The velocity range for the CO moment 0 map is from -2.0 to $+20.4 \mathrm{~km} \mathrm{~s}^{-1}$. The white contours range from $25 \%$ to $85 \%$ of the peak emission, in steps of $15 \%$. The peak of the continuum emission is $0.31 \mathrm{Jy}^{\text {beam }}{ }^{-1}$. The synthesized beam of the continuum image is shown in the lower right corner. The two diagonal dashed lines mark the places where the position-velocity diagrams, presented in Figure 2 , were obtained.

servations, made with the Submillimeter Array ${ }^{5}$ (SMA), of the young star DG Tau B and its associated outflow. These interferometric observations reveal velocity asymmetries about the flow axis over the entire length of the flow that can be interpreted as outflow rotation. Continuum and line observations of the circumstellar disk associated with DG Tau B are also presented. The paper is organized as follows: In $\$ 2$ and 3 we discuss the observations and results. In $\S 4$ we discuss the possible origin of rotating outflows, and in $\$ 5$ we present the conclusions.

\section{OBSERVATIONS}

The observations were carried out with the SMA on 2011 December and 2012 February, when the array was in its compact and extended configuration, respectively. The independent baselines in these configurations ranged in projected length from 10 to $140 \mathrm{k} \lambda$. The phase reference center for

\footnotetext{
5 The Submillimeter Array (SMA) is a joint project between the Smithsonian Astrophysical Observatory and the Academia Sinica Institute of Astronomy and Astrophysics, and is funded by the Smithsonian Institution and the Academia Sinica.
}

the observations was set to $\alpha_{J 2000.0}=04^{\mathrm{h}} 27^{\mathrm{m}} 02.66, \delta_{J 2000.0}$ $=+26^{\circ} 05^{\prime} 30^{\prime \prime}$. 4 . Two frequency bands, centered at 230.457 $\mathrm{GHz}$ (Upper Sideband) and 220.457 GHz (Lower Sideband) were observed simultaneously. We concatenated the two data sets using the task in MIRIAD called "uvcat". The two different observations were identical, and only the antenna configuration of the SMA changed. The primary beam of the SMA at $230 \mathrm{GHz}$ has a FWHM of 57", and the continuum and line emission arising from DG Tau B fall well within it.

The SMA digital correlator was configured to have 48 spectral windows ("chunks") of $104 \mathrm{MHz}$ and 128 channels each. This provided a spectral resolution of $0.812 \mathrm{MHz}(\sim 1 \mathrm{~km}$ $\mathrm{s}^{-1}$ ) per channel. However, some of these spectral windows included 512 channels (one of these bands included the CO line), providing $0.203 \mathrm{MHz}\left(\sim 0.26 \mathrm{~km} \mathrm{~s}^{-1}\right)$ of resolution. This very high spectral resolution allowed a reliable study of the CO kinematics.

Observations of Uranus provided the absolute scale for the flux density calibration. The gain calibrators were the quasars 3C 111 and 3C 84, while 3C 279 was used for bandpass calibration. The uncertainty in the flux scale is estimated to be be- 
tween 15 and $20 \%$, based on the SMA monitoring of quasars.

The data were calibrated using the IDL superset MIR, originally developed for the Owens Valley Radio Observatory (OVRO, Scoville et al. 1993) and adapted for the SMA. ${ }^{6}$ The calibrated data were imaged and analyzed in the standard manner using the MIRIAD (Sault et al. 1995) and KARMA (Gooch 1996) softwares ${ }^{7}$. A $1300 \mu \mathrm{m}$ continuum image was obtained by averaging line-free channels in the lower sideband with a bandwidth of about $4 \mathrm{GHz}$. A continuum image using both bands was also obtained, however similar results were reached. For the line emission, the continuum was also removed. We set the ROBUST parameter of the task INVERT to +2 to obtain a better sensitivity losing some angular resolution. The resulting r.m.s. noise for the continuum was about $9 \mathrm{mJy}$ beam $^{-1}$, at an angular resolution of 1 .' $^{\prime} 87 \times 11^{\prime \prime} 63$ with a P.A. $=64.3^{\circ}$. The r.m.s. noise in each channel of the spectral line data was about $90 \mathrm{mJy} \mathrm{beam}^{-1}$ at the same angular resolution.

\section{RESULTS}

In Figure 1, we present the resulting map of the ${ }^{12} \mathrm{CO}(2$ 1) line and $1300 \mu \mathrm{m}$ continuum observations made with the Submillimeter Array of DG Tau B. This image is overlaid with Hubble Space Telescope (HST) images obtained with the infrared camera WFPC2 (Stapelfeldt et al. 1997). Figure 1 reveals the innermost parts of the NW monopolar redshifted outflow with the rotated "V" morphology reported for the first time by Mitchell et al. (1997). By contrast, the blueshifted $\mathrm{CO}$ emission is confined to a compact region, close to the disk. The spatial correspondence between knots in the optical jet and successive broadenings of the outflow supports the hypothesis that the molecular flow is produced by the action of multiple working surfaces or the entrainment of the ambient gas (Mitchell et al. 1997). In this image, are also seen the optical jet and the infrared nebulosities of scattered light. The $1300 \mu \mathrm{m}$ emission falls very well within the circumstellar disk imaged in absorption by the broadband imaging of the HST. We conclude that the $1300 \mu \mathrm{m}$ emission here is tracing the innermost parts of the disk, very close to the young star DG Tau B.

For the continuum $1300 \mu \mathrm{m}$ emission, and using a Gaussian fitting, we found that the flux density and peak intensity values of DG Tau B are 590 $\pm 30 \mathrm{mJy}$ and $310 \pm 20 \mathrm{mJy} \mathrm{beam}^{-1}$, respectively. We also find from this fit that the source has a deconvolved size of 1 .' $8 \pm 0$." $5 \times 1$." $5 \pm 0$.' 6 with a P.A. $=$ $+30^{\circ} \pm 5^{\circ}$. Therefore, at the distance of the Taurus molecular cloud complex $(150 \mathrm{pc})$ the size of the continuum source is about $270 \times 225 \mathrm{AU}$.

Following Hildebrand (1983) and assuming optically thin isothermal dust emission, a gas-to-dust ratio of 100 , a dust temperature of $30 \mathrm{~K}$, a dust mass opacity $\kappa_{1300} \mu \mathrm{m}=1.1 \mathrm{~cm}^{2}$ $\mathrm{g}^{-1}$ (Ossenkopf \& Henning 1994), and that this object is located at $150 \mathrm{pc}$, we estimate the total mass associated with the dust continuum emission to be $0.08 \mathrm{M}_{\odot}$, this in a very good agreement with the value obtained by Guilloteau et al. (2011). The $1300 \mu \mathrm{m}$ continuum emission is thus tracing the circumstellar disk surrounding DG Tau B. Furthermore, we remark that the mass obtained here could be partially optically thick, and therefore this is a lower limit.

Following Zapata et al. (2014), assuming local thermody-

\footnotetext{
6 The MIR-IDL cookbook by C. Qi can be found at http://cfawww.harvard.edu/ cqi/mircook.html.

${ }^{7}$ The calibrated data can be obtained from: http://www.cfa.harvard.edu/
}

namic equilibrium (LTE), and that the ${ }^{12} \mathrm{CO}(2-1)$ molecular emission is optically thin, we estimate the outflow mass using the following equation:

$$
\frac{M\left(H_{2}\right)}{M_{\odot}}=6.3 \times 10^{-20} m\left(H_{2}\right) X_{\frac{H_{2}}{C O}}\left(\frac{c^{2} d^{2}}{2 k v^{2}}\right) \frac{\exp \left(\frac{5.5}{T_{e x}}\right) \int S_{v} d v \Delta \Omega}{\left(1-\exp \left[\frac{-11.0}{T_{e x}}\right]\right)},
$$

where all units are in cgs, $\mathrm{m}\left(\mathrm{H}_{2}\right)$ is mass of the molecular hydrogen, $\mathrm{X}_{\frac{\mathrm{H}_{2}}{\mathrm{CO}}}$ is the fractional abundance between the carbon monoxide and the molecular hydrogen $\left(10^{4}\right), c$ is the speed of light, $k$ is the Boltzmann constant, $v$ is the rest frequency of the $\mathrm{CO}$ line in $\mathrm{Hz}, d$ is distance $(150 \mathrm{pc}), S_{v}$ is the flux density of the $\mathrm{CO}$ (Jansky), $d v$ is the velocity range in $\mathrm{cm} \mathrm{s}^{-1}, \Delta \Omega$ is the solid angle of the source in steradians, and $\mathrm{T}_{e x}$ is excitation temperature taken to be $50 \mathrm{~K}$. We then estimate a mass for the outflow powered by DG Tau B of $3 \times 10^{-3} \mathrm{M}_{\odot}$. This value is consistent with the mass of other molecular outflows powered by young low-mass protostars, see Wu et al. (2004). The mass estimated here is only a lower limit because the CO emission is likely to be optically thick.

In Figure 2, we present two position-velocity diagrams or cuts obtained across the redshifted side of the outflow, named $\mathrm{C} 1$ and $\mathrm{C} 2$, see Figure 1. These diagrams reveal the spatial structure of the gas across the flow as a function of the radial velocities and at distances far from DG Tau B ( 1400 AU for the case of $\mathrm{C} 1$ and $1900 \mathrm{AU}$ for $\mathrm{C} 2$ ). In both cuts velocity gradients across the outflow with an amplitude of about $2 \mathrm{~km}$ $\mathrm{s}^{-1}$ are revealed. In the cut $\mathrm{C} 1$, the gradient is somewhat larger than $2 \mathrm{~km} \mathrm{~s}^{-1}$. This gradient is also confirmed by the spectra obtained in both sides of the flow as shown in Figure 2 in the lower panels. Both cuts were intentionally made over two optical bullets in order to reveal their dynamics (see Figure 1), however, only the bullet associated with the cut called C2 has a clear molecular counterpart. This is located in the middle of the borders of the molecular flow. This molecular emission associated with the optical bullet is too compact to reveal any velocity gradient.

In Figure 3 is presented the first moment or the intensity weighted velocity of the ${ }^{12} \mathrm{CO}(2-1)$ emission from the inner part of the outflow overlaid on the HST infrared image (using the spectral filter F675w) and with the SMA $1300 \mu m$ continuum image. The image reveals the dynamics of the $\mathrm{CO}$ gas in the innermost parts of the flow and in the vicinity of the optical jet. Here, the blue colors represent blueshifted emission, while the red colors, redshifted emission. The velocity gradient across the outflow is also revealed in this Figure, it is more evident in its blueshifted side and close to DG Tau B. A possibility of why the rotation is diluted farther from DG Tau B could come from the fact that the flow is not exactly on the plane of the sky, and far from the source the poloidal velocities dominate.

In Figure 3, in the lower panels, we also show two positionvelocity diagrams we made to reveal the kinematics of the molecular gas close to the circumstellar disk related with DG Tau B. In the position-velocity diagrams called D1 and D2 (at a distance from DG Tau B of about $220 \mathrm{AU}$ ), are revealed an asymmetric "V"-shaped (or, perhaps, a triangular shape) velocity pattern. The diagrams are made at a distance of about $250 \mathrm{AU}$ from the source. These velocity patterns have been attributed to outflow rotation (Lee et al. 2008; Zapata et al. 2010; Pech et al. 2012; Peters et al. 2014). These patterns are formed because the low-velocity gas far from the outflow axis has a velocity gradient (i.e. rotation), while the gas in the jet 

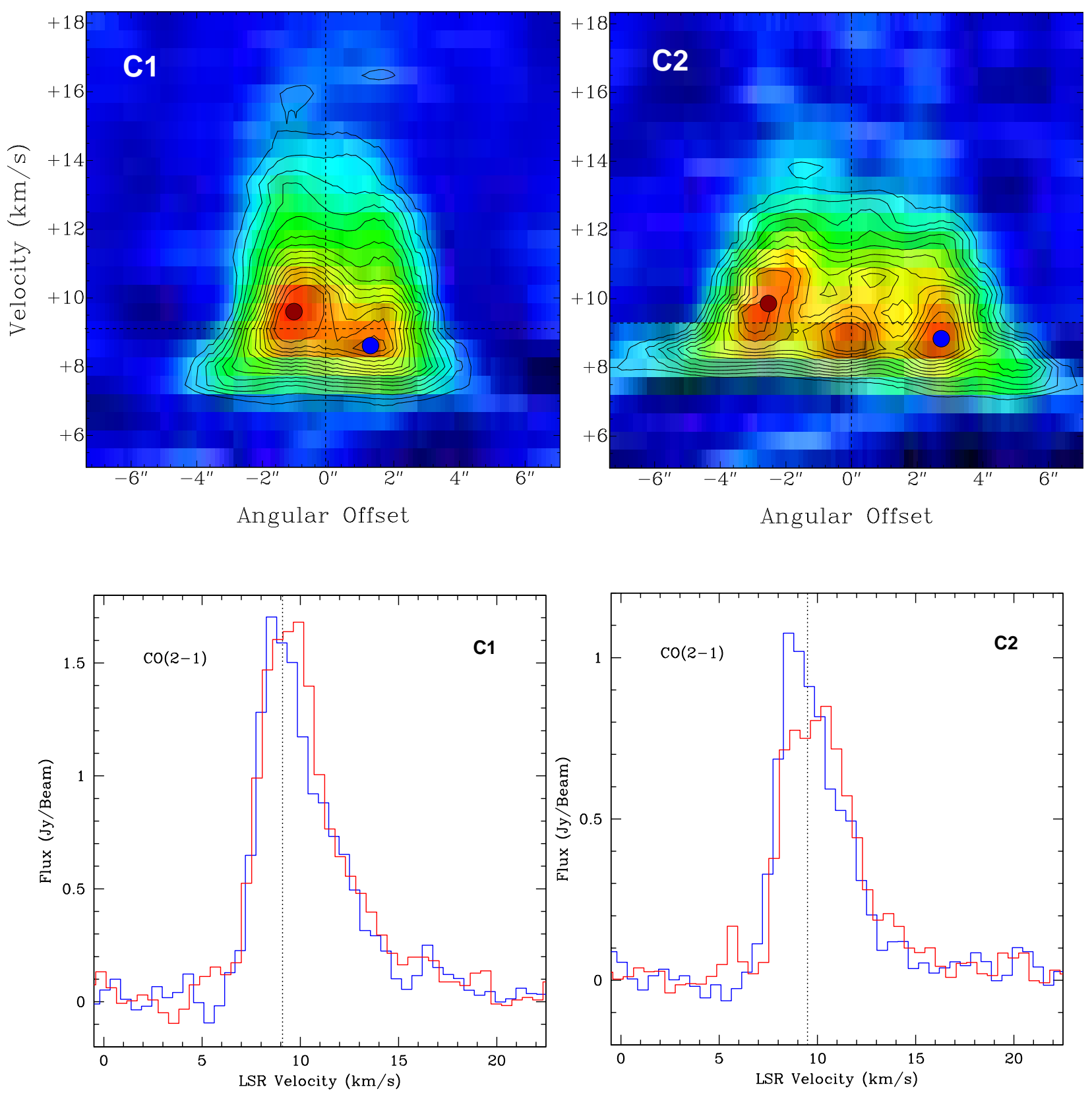

Figure 2. Upper images: Position-velocity relations across the redshifted side of the molecular outflow in DG Tau B: Radial velocity as function of on-the-sky distance. The angular offsets are in arcseconds and the LSR radial velocities in $\mathrm{km} \mathrm{s}^{-1}$. The dashed lines mark the position of the outflow axis and the median velocity of the gradient. The molecular gas material in the outflow at these positions has a radial velocity of about +9.0 to $+9.5 \mathrm{~km} \mathrm{~s}^{-1}$. The contours in $\mathrm{C} 1$ range from $25 \%$ to $90 \%$ of the peak emission, in steps of $5 \%$. The peak of the line emission is $1.9 \mathrm{Jy} \mathrm{beam}^{-1}$. For $\mathrm{C} 2$, the contours range from $40 \%$ to $90 \%$ of the peak emission, in steps of 5\%. The peak of the emission is $1.05 \mathrm{Jy} \mathrm{beam}^{-1}$. The synthesized beam is $1.87^{\prime \prime} \times 1.63^{\prime \prime}$ with a P.A. of $+64.3^{\circ}$, and the spectral resolution is $\sim 0.8 \mathrm{~km} \mathrm{~s}^{-1}$. The blue and red dots represent the positions where the spectra presented in the lower images were obtained. Lower images: CO spectra obtained in the positions shown in the upper images. The blue lines correspond to the spectra obtained in the blue dots, while the red lines to the red dots. The dashed lines mark the position of the median velocity of the gradient observed across the outflow.

and close to the axis seems to be only accelerated in direction of the flow. If the low-velocity gas were parallel to the direction of the outflow (i.e. no rotation), one would expect these $\mathrm{V}$ shapes to be symmetric with respect to the axis of the flow. The amplitude of such velocity asymmetries is about $4 \mathrm{~km}$ $\mathrm{s}^{-1}$. We noted that this gradient is larger to that observed far from the source, perhaps due to the conservation of angular momentum.

From ${ }^{12} \mathrm{CO}$ (and ${ }^{13} \mathrm{CO}$ ) observations one can obtain the disk mass and kinematics (T. Bourke et al. in preparation). The disk mass is $\sim 0.1 M_{\odot}$. Position-velocity diagrams of both isotopes show disk rotation in the same sense as the observed outflow rotation. These diagrams can be fitted by keplerian curves that give the central stellar mass as a function of inclination angle. The best fit gives $M_{*} \sim 0.5 M_{\odot}$ for an inclination angle of $65^{\circ}$.

\section{DISCUSSION}



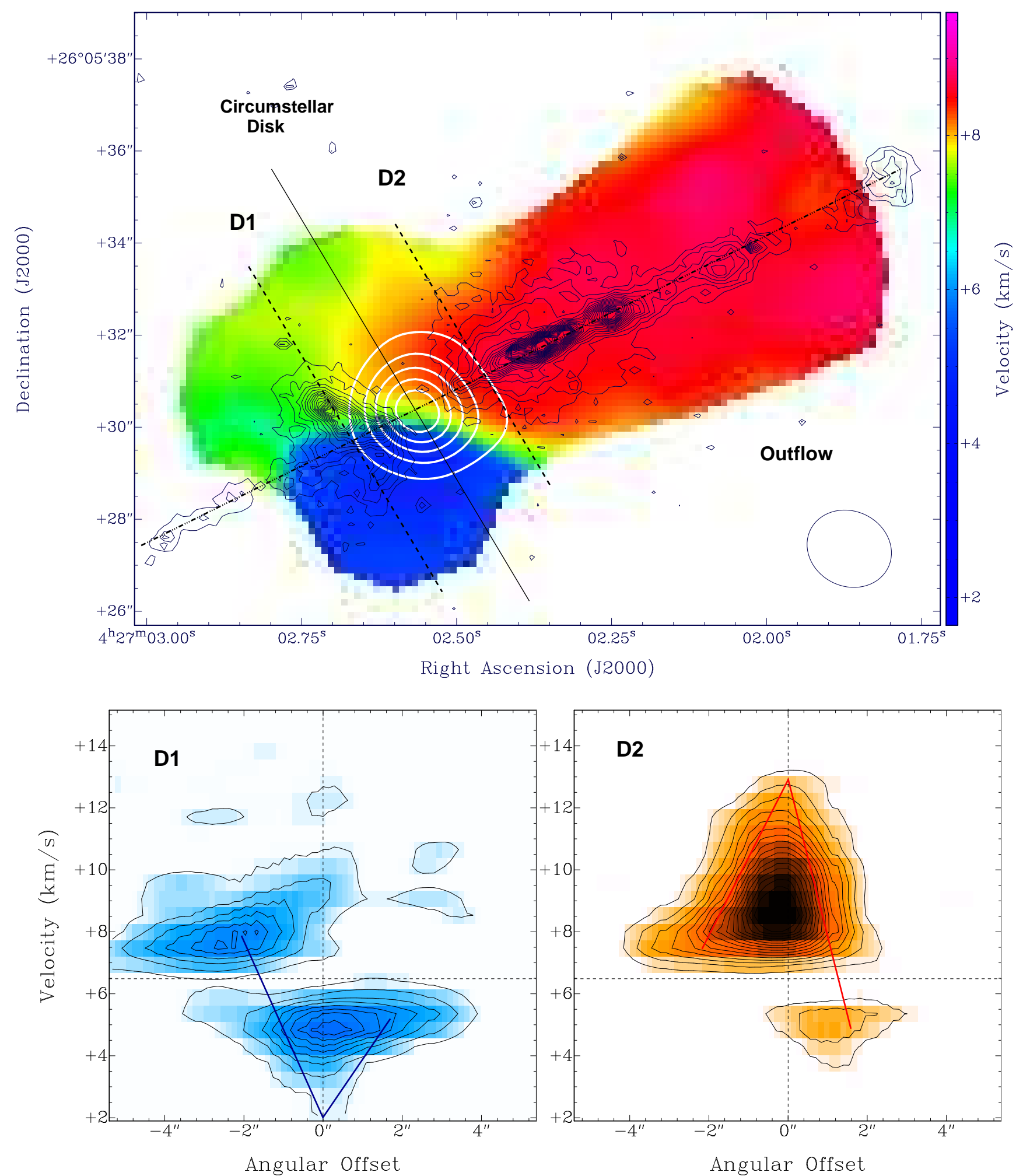

Figure 3. Upper image: SMA first moment or the intensity weighted velocity of the CO emission from the outflow overlaid in black contours with the HST infrared image (using the spectral filter F675w) and in white contours with the SMA $1300 \mu m$ continuum image. The white contours range from 25\% to $85 \%$ of the peak emission, in steps of $15 \%$. The peak of the continuum emission is $0.31 \mathrm{Jy} \mathrm{beam}^{-1}$. The synthesized beam of the continuum image is shown in the lower right corner. The diagonal dashed lines mark the places where the position-velocity diagrams, presented here, were made. The color-scale bar on the right indicate the LSR velocities in $\mathrm{km} \mathrm{s}^{-1}$. The continuous and dashed lines mark the disk (P.A. $=208.1^{\circ}$ ) and the outflow $\left(\right.$ P.A. $\left.=298.1^{\circ}\right)$ axis, respectively, and the positions of the cuts D1 and D2. Lower image: Position-velocity relations of the CO across the redshifted/blueshifted sides of the molecular outflow in DG Tau B, see upper image. The angular offsets are in arcseconds and the radial velocities in $\mathrm{km} \mathrm{s}^{-1}$. The dashed lines mark the position of the outflow axis and the median velocity of the gradient. The contours in D1 range from $20 \%$ to $90 \%$ of the peak emission, in steps of $10 \%$. The peak of the line emission is $0.8 \mathrm{Jy}$ beam $^{-1}$. For D2, the contours range from $40 \%$ to $90 \%$ of the peak emission, in steps of $5 \%$. The peak of the emission is $1.8 \mathrm{Jy}^{-1}$ beam ${ }^{-1}$. The synthesized beam is $1.87^{\prime \prime} \times 1.63^{\prime \prime}$ with a P.A. of $+64.3^{\circ}$, and the spectral resolution is $\sim 0.8 \mathrm{~km} \mathrm{~s}^{-1}$. The red and blue lines in the lower panels mark the position of the jet and the velocity gradients. 


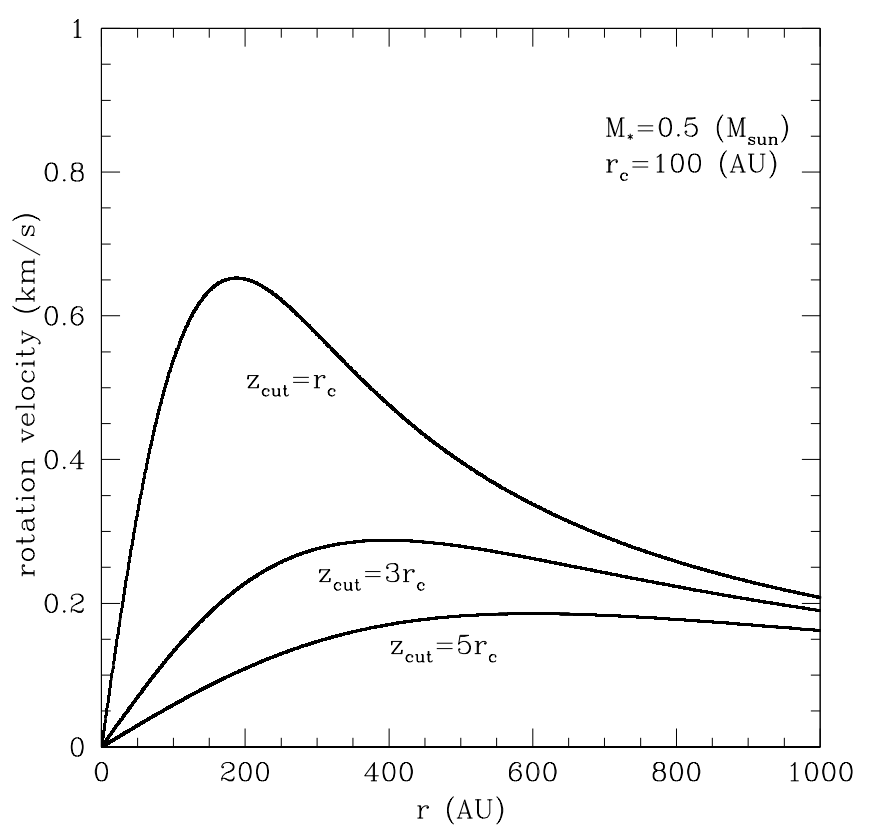

Figure 4. Rotation velocity as function of the distance to the rotation axis for a rotating collapsing isothermal flow for cuts parallel to the mid plane at different heights above the disk, measure in units of the centrifugal radius, $r_{c}$ $=100 \mathrm{AU}$.

\subsection{Outflow rotation}

For this discussion, the observed velocity gradient across and in the entire length of the molecular outflow from DG Tau B is interpreted as rotation. Even though, as discussed in Launhardt et al. (2009); Zapata et al. (2010); Pech et al. (2012) there might be some alternative explanations for the observed gradient (e.g. Soker 2005; Cerqueira et al. 2006), however, these explanations are unlikely in the case of DG Tau B. For example, precession or the presence of a binary outflow seem to be ruled out by the existence of the collimated and straight optical jet imaged by the HST in the middle of the molecular outflow (see Figure 1). Also, infrared images obtained by McGroarty \& Ray (2004) at parsec scales show no evidence of precession for the optical jet. The model proposed by Cerqueira et al. (2006) is discarded in the case of DG TAU B because the optical jet is not preccesing and the velocity gradient is observed in the outflow.

\subsection{On the origin of the molecular flow rotation}

From Figures 2 and 3, the observed $\mathrm{CO}$ outflow has a radial velocity of $v_{r} \sim 3 \mathrm{~km} \mathrm{~s}^{-1}$ (subtracting the LSR velocity of central source), and a rotation speed $v_{\varphi} \sim 1 \mathrm{~km} \mathrm{~s}^{-1}$ at a distance from the jet axis $R \sim 150$ AU. As discussed above, the outflow rotation is in the same direction as the rotation of the keplerian disk around the central star. Also, given the inclination angle of the jet axis with respect to the 1.o.s. $\theta_{i} \sim 65^{\circ}$ (Eislöffel \& Mundt 1998), the corrected poloidal speed is $v_{p}=v_{r} / \cos \left(\theta_{i}\right) \sim 7.1 \mathrm{~km} \mathrm{~s}^{-1}$.

What is the origin of this rotating molecular flow? One possibility is that the observed molecular flow is infalling rotating gas from the parent molecular core. In particular, one can test the collapse flow solution of a rotating isothermal core TSC (Terebey et al. 1984). This flow will collapse to the mid plane and will form a disk with a size given by the centrifugal radius, $r_{c}=\Omega_{c}^{2} G^{3} M_{*}^{3} / 16 a^{8}$, where $\Omega_{c}$ is the rotation rate of the core, $G$ is the gravitational constant, $a$ is the sound speed, and
$M_{*}$ is the mass of the central star. Figure 4 shows the rotation velocity of the collapsing flow as a function of the distance to the rotation axis along cuts parallel to the disk midplane, at different heights from the disk: $z=r_{c}, 3 r_{c}, 5 r_{c}$, where we assumed a centrifugal radius $r_{c}=100 \mathrm{AU}$. This figure shows that for all the curves the rotation velocity of the collapsing gas is smaller than the observed rotation speed $v_{\varphi} \sim 1 \mathrm{~km} \mathrm{~s}^{-1}$. In fact, for the cut at $z=5 r_{c}=500 \mathrm{AU}$, the rotational speed of the infalling gas is $v_{\phi}<0.2 \mathrm{~km} \mathrm{~s}^{-1}$; this velocity decreases even more for cuts further up from the disk midplane. Therefore, the observed rotating flow cannot correspond to infalling material from this type of rotating envelope.

Another possibility is that the rotating molecular flow corresponds to a slow wind ejected from the keplerian disk. We now examine two types of disk winds: magneto-centrifugal winds and photoevaporated winds.

For a magneto-centrifugal wind, the poloidal $v_{p}$ and rotation $v_{\phi}$ velocities at a distance $R$ from the rotation axis, can be related to the keplerian rotation rate at the footpoint $r_{0}$ on the disk, $\Omega_{K 0}=\left(G M_{*} / r_{0}^{3}\right)^{1 / 2}$, by a cubic equation (eq. (4) of Anderson et al. 2003). For a central star with a mass $M_{*}=0.5 M_{\odot}$, this equation gives an footpoint $r_{0} \sim 11 \mathrm{AU}$. One can also obtain the parameter $\lambda_{\phi}=R v_{\phi} /\left(r_{0}^{2} \Omega_{K 0}\right) \sim 2.1$, which is the ratio of the specific angular momentum at $R$ to the specific angular momentum at the footpoint $r_{0}$. When the jets reach high Alvénic Mach numbers and large radii, $\lambda_{\phi}$ should be equal to the lever arm, $\lambda=\left(r_{A} / r_{0}\right)^{2}$, where $r_{A}$ is the Alfvén radius where the flow reaches the Alfvén speed (e.g., Ferreira et al. 2006). The lever arm of the flow can be estimated in the asymptotic regime from the ratio of the poloidal speed to the kepkerian speed at the footpoint, $r_{A} / r_{0} \sim v p / 2^{1 / 2} v_{K}\left(r_{0}\right) \sim 0.8$. Then, the implied values of $\lambda_{\phi}$ and $\lambda$ are very small, with values of $\sim 1-2$, providing very little poloidal acceleration. Furthermore, since the lever arm relates the wind mass-loss rate with the disk accretion rate, $\dot{M}_{w} \sim \dot{M}_{\text {acc }} / \lambda$, these low values of $\lambda$ imply a very high efficiency of mass ejection. In contrast, values of $\lambda \sim 10$ are expected for MHD disk winds models such that the wind carries away one tenth of the mass accretion rate (e.g., Königl \& Pudritz 2000). In summary, because the molecular flow has a poloidal velocity comparable to the rotation speed, its interpretation as a magneto-centrifugal disk wind poses severe problems of mass ejection.

The second type of disk winds are photoevaporated winds that arise because the gas at the disk surface is heated by high energy photons from the central star and is able to escape from the local gravitational potential well. Models of these winds from disks around low mass stars include mainly heating by FUV and X rays (e.g. Font et al. 2004; Gorti \& Hollenbach 2009; Owen et al. 2011). These flows have low poloidal speeds $v_{p} \sim$ few $\times a$, where the thermal speed is $a \sim 2 \mathrm{~km} \mathrm{~s}^{-1}\left(T / 10^{3} K\right)^{1 / 2}(\mu / 2)^{-1 / 2}$, where $T$ is the gas temperature, and $\mu$ is the mean mass per particle in units of the hydrogen mass $m_{H}$ (e.g., Lugo et al. 2004). A characteristic boundary where a photoevaporated wind can escape is given by the gravitational radius $r_{g}=G M_{*} / a^{2}$, although pressure gradients can allow photoevaporation from smaller radii, $r \sim r_{g} / 3-r_{g} / 2$. This radius can be written as $r_{g} \sim 108\left(T / 10^{3} K\right)^{-1}(\mu / 2)\left(M_{*} / 0.5 M_{\odot}\right)$ AU. Photoevaporated winds are thermal winds and evolve conserving the specific angular momentum injected at the disk footpoint, i.e., $r_{0}^{2} \Omega_{K 0}=R v_{\varphi}$. For the observed specific angular momentum, this relation gives an ejection point $r_{0} \sim 51 \mathrm{AU} \sim r_{g} / 2$. 
On the other hand, we will now show that magnetocentrifugal and photoevaporated slow disk winds do not have enough linear or angular momentum to account for the observed rates in the molecular outflow. The observed molecular flow has a mass $M_{H_{2}} \sim 10^{-3} M_{\odot}$, and the associated dynamical time is $t_{\text {dyn }}=1000 \mathrm{AU} / v_{p} \sim 880 \mathrm{yr}$. Then, the molecular outflow mass-loss rate is $\dot{M}_{\text {outflow }} \sim 1.1 \times 10^{-6} M_{\odot} \mathrm{yr}^{-1}$, its momentum rate is $\dot{P}_{\text {outflow }}=\dot{M}_{\text {outflow }} v_{p} \sim 6 \times 10^{-6} M_{\odot} \mathrm{yr}^{-1} \mathrm{~km} \mathrm{~s}^{-1}$, and its angular momentum rate is $\dot{L}_{\text {outflow }}=\dot{M}_{\text {outflow }} R v_{\phi} \sim$ $1.65 \times 10^{-4} M_{\odot} \mathrm{AU} \mathrm{km} \mathrm{s}{ }^{-1}$. This linear momentum rate is similar to the rate measured by Mitchell et al. (1994) and, correcting for partial ionization, is consistent to the momentum rate of the high velocity atomic jet (Podio et al. 2011). Instead, for the slow disk winds considered here, assuming a mass-loss rates similar to that of the central atomic jet $\dot{M}_{w} \sim$ $10^{-8} M_{\odot} \mathrm{yr}^{-1}$, the linear momentum rate is $\dot{P}_{w}=\dot{M}_{w} v_{p} \sim$ $5.5 \times 10^{-8} M_{\odot} \mathrm{yr}^{-1} \mathrm{~km} \mathrm{~s}^{-1}$, and the angular momentum rate is $\dot{L}_{w}=\dot{M}_{w} r_{0} v_{K 0} \lambda_{\phi}=\dot{M}_{w} r v_{\phi}=1.5 \times 10^{-6} M_{\odot} \mathrm{AU} \mathrm{yr}^{-1} \mathrm{~km} \mathrm{~s}^{-1}$. Both rates are smaller than the molecular outflow rates by the factor $\dot{M}_{\text {outflow }} / M_{w} \sim 100$.

This large discrepancy between the slow disk wind rates and the outflow rates indicates that the molecular outflow is probably material from a rotating parent cloud entrained by a fast wind (even though the TSC infalling envelope has rotational speeds smaller than the observed $v_{\phi}$ ). It would be important to show that such entrainment process is capable of carrying also the observed angular momentum (e.g., Canto et al. 1996). A detailed modeling of this process is a very interesting problem but is out of the scope of this paper.

\section{CONCLUSIONS}

We have reported ${ }^{12} \mathrm{CO}(2-1)$ line observation of DG Tau B, and discovered velocity asymmetries about the flow axis with an amplitude roughly on the order of $2 \mathrm{~km} \mathrm{~s}^{-1}$ far from its exciting source and about $4 \mathrm{~km} \mathrm{~s}^{-1}$ close to it. This difference in velocities might be due to the conservation of the angular momentum. Similar velocity asymmetries are found on both sides of the outflow, and we interpret them as evidence for outflow rotation. The outflow rotation sense is identical to that revealed in the circumstellar disk. We show that slow disk winds ejected at distances of tens of AU from the star would have the observed poloidal and rotation velocities. Nevertheless, the observed linear and angular momentum rates of the outflow are too large compared to the rates of these slow disk winds and indicate that the molecular rotating flow is probably entrained material form the parent core. DG Tau B appears to be a promising laboratory for future studies of this entrainment process.

L.A.Z, S. L., L. F. R., L.L., and D.T. acknowledge the financial support from DGAPA, UNAM, and CONACyT, México. Facilities: The Submillimeter Array (SMA)

\section{REFERENCES}

Alexander, R. D., Clarke, C. J., \& Pringle, J. E. 2005, MNRAS, 358, 283
Anderson, J. M., Li, Z.-Y., Krasnopolsky, R., \& Blandford, R. D. 2003, ApJ, 590, L107

Arce, H. G., Shepherd, D., Gueth, F., et al. 2007, Protostars and Planets V, 245

Canto, J., Raga, A. C.

\& Wilkin, F. P. 1996, ApJ, 469, 729

Cerqueira, A. H., Velázquez, P. F., Raga, A. C., Vasconcelos, M. J., \& de Colle, F. 2006, A\&A, 448, 231

Coffey, D., Bacciotti, F., Ray, T. P., Eislöffel, J., \& Woitas, J. 2007, ApJ, 663, 350

Eislöffel, J., \& Mundt, R. 1998, AJ, 115, 1554

Fendt, C. 2011, ApJ, 737, 43

Ferreira, J., Dougados, C., \& Cabrit, S. 2006, A\&A, 453, 785

Font, A. S., McCarthy, I. G., Johnstone, D., \& Ballantyne, D. R. 2004, ApJ, 607,890

Gooch, R. 1996, Astronomical Data Analysis Software and Systems V, 101, 80

Gorti, U., \& Hollenbach, D. 2009, ApJ, 690, 1539

Gorti, U., Hollenbach, D., Najita, J., \& Pascucci, I. 2011, ApJ, 735, 90

Gramajo, L. V., Whitney, B. A., Gómez, M., \& Robitaille, T. P. 2010, AJ, 139,2504

Guilloteau, S., Dutrey, A., Piétu, V., \& Boehler, Y. 2011, A\&A, 529, A105

Hildebrand, R. H. 1983, Quarterly Journal of the Royal Astronomical

Society, 24, 267

Jones, B. F., \& Cohen, M. 1986, ApJ, 311, L23

Klaassen, P. D., Juhasz, A., Mathews, G. S., et al. 2013, A\&A, 555, A73

Königl, A., \& Pudritz, R. E. 2000, Protostars and Planets IV, 759

Launhardt, R., Pavlyuchenkov, Y., Gueth, F., et al. 2009, A\&A, 494, 147

Lee, C.-F., Ho, P. T. P., Bourke, T. L., et al. 2008, ApJ, 685, 1026

Li, Z.-Y., \& Shu, F. H. 1996, ApJ, 468, 261

Lugo, J., Lizano, S., \& Garay, G. 2004, ApJ, 614, 807

Luhman, K. L., Allen, P. R., Espaillat, C., Hartmann, L., \& Calvet, N. 2010, ApJS, 186, 111

McGroarty, F. \& Ray, T. P. 2004, A\&A, 420, 975

McKee, C. F., \& Ostriker, E. C. 2007, ARA\&A, 45, 565

Mitchell, G. F., Hasegawa, T. I., Dent, W. R. F., \& Matthews, H. E. 1994, ApJ, 436, L177

Mitchell, G. F., Sargent, A. I., \& Mannings, V. 1997, ApJ, 483, L127

Mundt, R., \& Fried, J. W. 1983, ApJ, 274, L83

Ossenkopf, V., \& Henning, T. 1994, A\&A, 291, 943

Owen, J. E., Ercolano, B., \& Clarke, C. J. 2011, MNRAS, 412, 13

Padgett, D. L., Brandner, W., Stapelfeldt, K. R., et al. 1999, AJ, 117, 1490

Pech, G., Zapata, L. A., Loinard, L., \& Rodríguez, L. F. 2012, ApJ, 751, 78

Peters, T., Klaassen, P. D., Seifried, D., Banerjee, R., \& Klessen, R. S. 2014, MNRAS, 437, 2901

Podio, L., Eislöffel, J., Melnikov, S., Hodapp, K. W., \& Bacciotti, F. 2011, A\&A, 527, A13

Pudritz, R. E., Ouyed, R., Fendt, C., \& Brandenburg, A. 2007, Protostars and Planets V, 277

Rodriguez, L. F., Anglada, G., \& Raga, A. 1995, ApJ, 454, L149

Rodríguez, L. F., Dzib, S. A., Loinard, L., et al. 2012, RMA\&A, 48, 243

Sault, R. J., Teuben, P. J., \& Wright, M. C. H. 1995, Astronomical Data Analysis Software and Systems IV, 77, 433

Scoville, N. Z., Carlstrom, J. E., Chandler, C. J., et al. 1993, PASP, 105, 1482

Shang, H., Li, Z.-Y., \& Hirano, N. 2007, Protostars and Planets V, 261

Shu, F. H., Najita, J., Ostriker, E. C., \& Shang, H. 1995, ApJ, 455, L155

Shu, F. H., Najita, J. R., Shang, H., \& Li, Z.-Y. 2000, Protostars and Planets IV, 789

Soker, N. 2005, A\&A, 435, 125

Stapelfeldt, K., Burrows, C. J., Krist, J. E., \& WFPC2 Science Team 1997, Herbig-Haro Flows and the Birth of Stars, 182, 355

Terebey, S., Shu, F. H., \& Cassen, P. 1984, ApJ, 286, 529

Torres, R. M., Loinard, L., Mioduszewski, A. J., \& Rodríguez, L. F. 2009, ApJ, 698, 242

Wu, Y., Wei, Y., Zhao, M., et al. 2004, A\&A, 426, 503

Zapata, L. A., Schmid-Burgk, J., Muders, D., et al. 2010, A\&A, 510, A2

Zapata, L. A., Arce, H. G., Brassfield, E., et al. 2014, MNRAS, 441, 3696 